Improved coupling of Josephson junction arrays to the open space

F. Song (宋凤斌), M. He (何明), M. I. Faley, L. Fang (方兰), and A. M. Klushin

Citation: Journal of Applied Physics 108, 063903 (2010);

View online: https://doi.org/10.1063/1.3482023

View Table of Contents: http://aip.scitation.org/toc/jap/108/6

Published by the American Institute of Physics

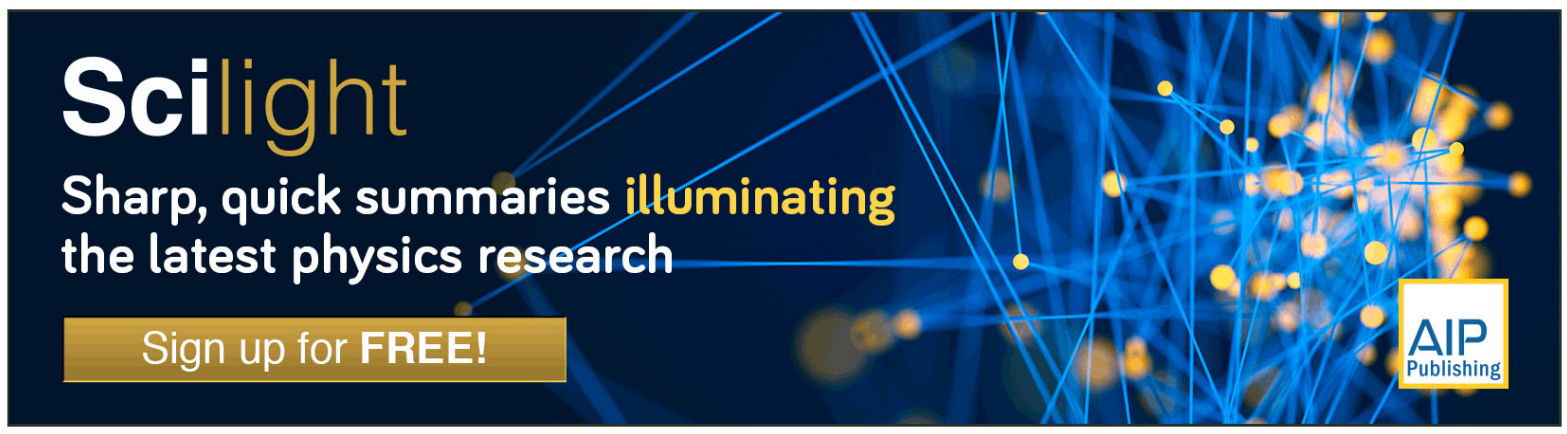




\title{
Improved coupling of Josephson junction arrays to the open space
}

\author{
F. Song (宋凤斌), ${ }^{1,2}$ M. He (何明), ${ }^{2}$ M. I. Faley, ${ }^{3}$ L. Fang (方兰), ${ }^{2}$ and A. M. Klushin ${ }^{1, a)}$ \\ ${ }^{1}$ Institute of Bio- and Nanosystems and JARA-Fundamentals of Future Information Technology, \\ Forschungszentrum Jülich, D-52425 Jülich, Germany \\ ${ }^{2}$ Department of Electronics, Nankai University, 300071 Tianjin, People's Republic of China \\ ${ }^{3}$ Institute of Solid State Research and JARA-Fundamental of Future Information Technology, \\ Forschungszentrum Jülich, D-52425 Jülich, Germany
}

(Received 18 June 2010; accepted 26 July 2010; published online 17 September 2010)

\begin{abstract}
The substrates of Josephson junctions have recently been considered as dielectric resonator antennas in order to increase self-emission power from the junctions. Further investigations and interesting phenomena demonstrating this mechanism are presented in this paper. Junction arrays of the same layout were located on various substrates, and Shapiro steps and self-emission were studied in the course of the experiments and simulations. It is demonstrated that suitable substrates can be utilized to improve the coupling of the junctions to the open space for both irradiation and self-emission at the same time. (C) 2010 American Institute of Physics. [doi:10.1063/1.3482023]
\end{abstract}

\section{INTRODUCTION}

The coupling of Josephson junctions to the open space has been discussed for almost half a century. However, it is still a challenging topic due to the unacceptable impedance mismatching between the open space and a single junction whose intrinsic impedance is rather small. ${ }^{1}$ Coherent Josephson junction arrays have been found to be a promising solution for this challenge. ${ }^{2}$ In any case, external antennas should be combined to further moderate this impedance mismatching.

During the development of modern wireless communications, two classes of novel antennas have been extensively investigated and described, i.e., the microstrip patch antenna and the dielectric resonator antenna. Compared with microstrip antennas, dielectric resonator antennas are more suitable for working at higher frequencies, especially for millimeter and submillimeter wavelengths. That is because, at these frequencies, the conductor loss of metallic antennas becomes severe and the efficiency of the antennas is reduced significantly. $^{3}$

In the meantime, the combination of Josephson junction arrays and microstrip patch antennas has been studied for about two decades. ${ }^{2,4-6}$ Nevertheless, utilization of the dielectric resonator antenna to decrease impedance mismatching is not as extensive as use of the microstrip antenna. We recently reported that enhanced self-emission from niobium junctions ${ }^{7}$ and bicrystal high-temperature superconductor junctions ${ }^{8}$ was measured with the assistance of intrinsic resonances of their own substrates. In this case, the substrates were considered to be dielectric resonator antennas, which deliver the self-emission power to the open space.

Furthermore, by this method, not only self-emission of the junctions but also external irradiation on the junctions can be optimized. As is well known, Josephson junctions arrays can be developed for use in voltage standards by utilizing the Shapiro steps under external irradiation. ${ }^{9,10}$ The

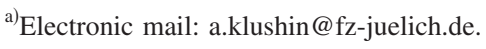

key point is that the parameters of junctions and the irradiation coupled into the junctions should be uniform enough to realize the coherence of junctions inside an array. In the following, we show that the coupling of all the junctions in a series array to the open space could be improved homogeneously by choosing a suitable substrate as the dielectric resonator antenna.

\section{EXPERIMENT}

The arrangement of our measurement system was shown in Fig. 1, which has been published elsewhere. ${ }^{7,8}$ To detect self-emission from the Josephson junctions, the measuring set-up was exactly the same as that shown in this figure. For the observations of the Shapiro steps of the junction arrays, external irradiation in the frequency range from $68 \mathrm{GHz}$ to $78 \mathrm{GHz}$ was directly fed into the quasioptical resonator through standard WR-12 waveguides. The quasioptical resonator consisted of a horn antenna and a plane mirror. Electromagnetic field distribution inside this resonator was simu-

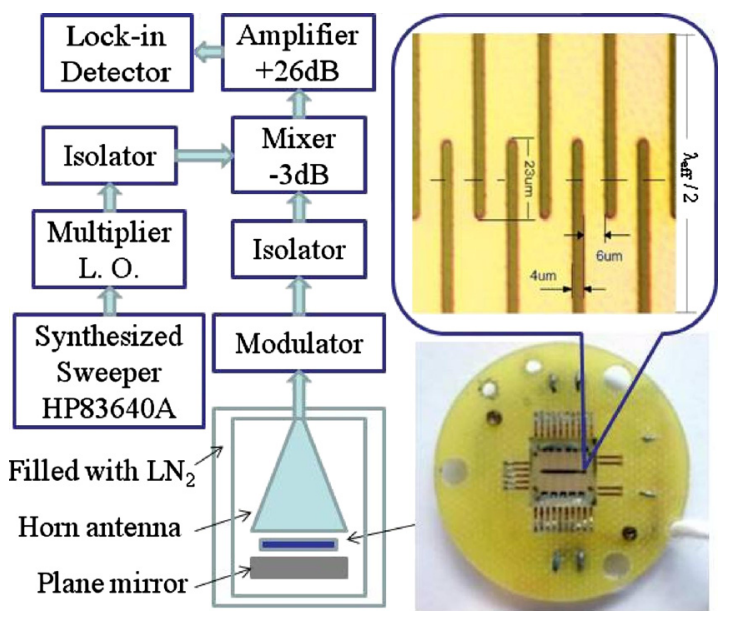

FIG. 1. (Color online) Schematic of measurement system and photos of the sample. The blow-up shows an enlarged fragment of the array of hightemperature superconductor Josephson junctions. 


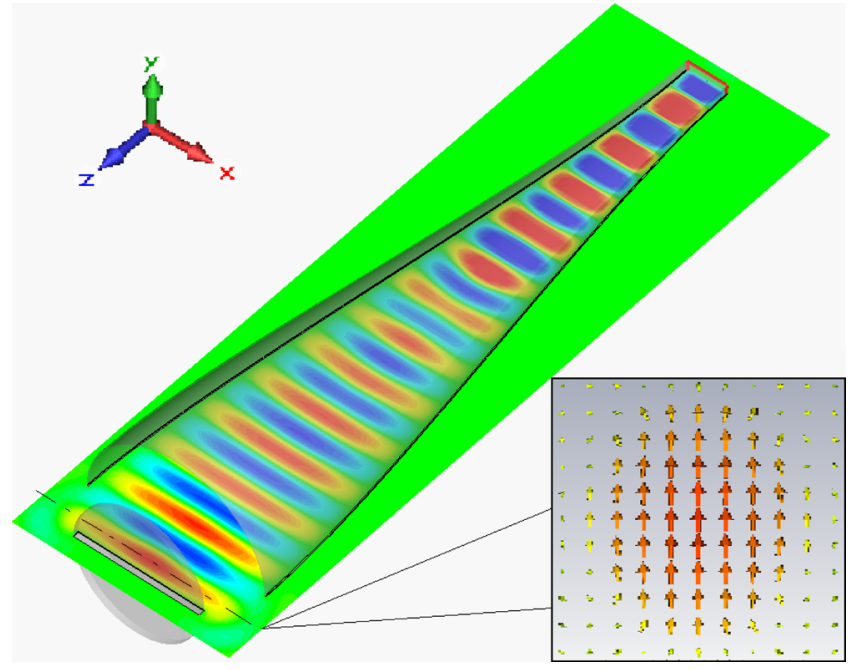

FIG. 2. (Color online) Distribution of electric field intensity in the quasioptical resonator including a horn antenna and a plane mirror. The standing wave was formed at some resonant frequencies which could be adjusted by the distance of the horn antenna and the plane mirror. The inset shows the uniform electric field distribution on the cross section of the antinodes.

lated by utilizing the CST microwave studio software, as shown in Fig. 2.

A standing wave was formed with a series of electric field antinodes at fixed positions inside the resonator. The amplitude of electric field intensity reached a maximum at the center of these antinodes. From the cross section view (the inset of Fig. 1), electric field was considered to be uniform with the same polarization in rather large area, compared with the dimensions of our samples. The distance between the horn antenna and the plane mirror should be adjusted for different frequencies of the irradiation to shape the standing wave. The sample was located around the antinodes in order to couple the intrinsic resonance of the substrate into the standing wave. ${ }^{7,8}$ In this way, the transmission of electromagnetic field power between the Josephson junctions and the open space becomes easier, i.e., the impedance coupling is improved.

To experimentally confirm the influence of the substrate on the coupling of Josephson junctions to the open space, the junction arrays of the same design as before ${ }^{8}$ were located on various substrates. The layout is also shown in Fig. 1. There were two kinds of substrate with different materials, namely magnesium oxide $(\mathrm{MgO})$ and yttrium-stabilized zirconium (YSZ). Their dielectric constants were $\varepsilon_{\mathrm{r}}=10$ and $\varepsilon_{\mathrm{r}}=26$, respectively. The fabrication of thin films of $\mathrm{YBa}_{2} \mathrm{Cu}_{3} \mathrm{O}_{7}$ on $\mathrm{MgO}$ substrates was described previously. ${ }^{11}$ The fabrication of shunted bicrystal junctions on these substrates was similar to the procedure published elsewhere in detail. ${ }^{12}$ The dimensions of these substrates were $10 \times 10 \mathrm{~mm}^{2}$, but with different thicknesses ranging from 0.3 to $1.0 \mathrm{~mm}$. Moreover, two additional thin substrates were used with dimensions of 10 $\times 10 \times 0.1 \mathrm{~mm}^{3}$, whose the materials were $\mathrm{MgO}$ and lanthanum aluminum oxide $(\mathrm{LaAlO})$ with $\varepsilon_{\mathrm{r}}=25$. They were attached to the rear of the original substrate of each sample. The performance of the various substrates for the coupling was explored, by measuring the Shapiro steps and selfemission from about 68 to $78 \mathrm{GHz}$ of the series junction arrays.
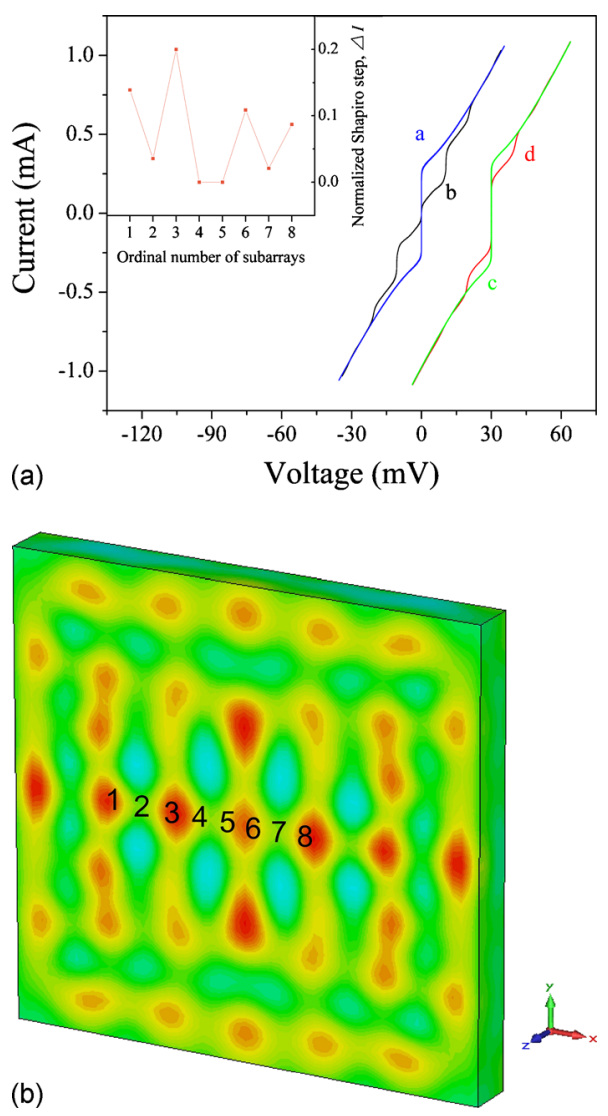

FIG. 3. (Color online) (a) Measurements of the junction array located on the $\mathrm{MgO}$ substrate. The $I-V$ curve of the third subarray without irradiation (curve a); the $I-V$ curve of the third subarray under irradiation at $76.10 \mathrm{GHz}$ (curve b); the $I-V$ curve of the fourth subarray without irradiation (curve c); the $I-V$ curve of the fourth subarray under irradiation at $76.10 \mathrm{GHz}$ (curve d). The normalized Shapiro steps $\Delta I$ of different subarrays were exhibited in the inset. (b) Distribution of electric field intensity on the $\mathrm{MgO}$ substrate shows the nonuniform irradiation power for different subarrays along the grain boundary. The position of each subarray is marked by its order number.

\section{MEASUREMENTS AND SIMULATIONS}

\section{A. Magnesium oxide}

Our investigation was motivated by measurements of Shapiro steps of the junction array located on the $10 \times 10$ $\times 1.0 \mathrm{MgO}$ substrate. The series array contains eight subarrays of 67 junctions each, which could be measured separately by special thin-film superconductor strips. An interesting phenomenon was revealed when the sample was exposed under external irradiation. The optimum coupling of the junctions to the standing wave inside the quasioptical resonators was attained by adjusting several parameters as before, ${ }^{8}$ namely the frequency $f_{\mathrm{r}}$ and the amplitude of the external radiation power source, the distance between the plane mirror and our sample, the position of the horn antenna and the angle $\alpha$ between the grid and the electric field $\boldsymbol{E}$ of the external radiation in the waveguide. However, under irradiation at $76.10 \mathrm{GHz}$ the Shapiro steps of each subarray were extremely nonuniform as shown in the inset of Fig. 3(a). To exclude the variances in parameters of different subarrays, the normalized Shapiro step $\Delta I=\Delta I_{1} / I_{\mathrm{c}}$ was introduced, where $I_{\mathrm{c}}$ is the critical current of the subarray and $\Delta I_{1}$ is the height of its first Shapiro step. The inset shows that the 
TABLE I. Significant measurement records of the Shapiro steps and self-emission of the Josephson junction arrays of 536 junctions located on the YSZ substrates with different thicknesses and attached thin substrates.

\begin{tabular}{|c|c|c|c|c|c|}
\hline \multirow{2}{*}{$\begin{array}{l}\text { Thickness of } \\
\text { the YSZ substrates } \\
(\mathrm{mm})\end{array}$} & \multicolumn{2}{|c|}{ Attached thin substrates } & \multicolumn{2}{|c|}{ The optimum first Shapiro step } & \multirow[b]{2}{*}{$\begin{array}{l}\text { Max. emission power } \\
\text { (a.u.) }\end{array}$} \\
\hline & Materials & Thickness & $\begin{array}{l}\text { Frequency } \\
\quad(\mathrm{GHz})\end{array}$ & $\begin{array}{l}\text { Voltage } \\
(\mathrm{mV})\end{array}$ & \\
\hline 0.5 & \multicolumn{2}{|c|}{ None } & 77.09 & 85.06 & 4.13 \\
\hline 0.3 & \multicolumn{2}{|c|}{ None } & 68.98 & 46.92 & 0.42 \\
\hline 0.3 & $\mathrm{MgO}$ & $0.1 \mathrm{~mm}$ & 75.29 & 61.49 & 0.95 \\
\hline \multirow[t]{3}{*}{0.3} & $\mathrm{LaAlO}$ & $0.1 \mathrm{~mm}$ & 72.85 & 69.56 & 4.65 \\
\hline & & & 75.00 & 82.16 & \\
\hline & & & 77.10 & 84.29 & \\
\hline
\end{tabular}

values $\Delta I$ of the eight subarrays strongly depended on the position of each subarray along the grain boundary. For example, in Fig. 3(a) the current-voltage $(I-V)$ curves of the third and the fourth subarrays with and without irradiation, respectively, illustrated a remarkable contrast. This reflects the widely differing coupling of the external irradiation to each subarray.

Nevertheless, it agreed with the simulation of the electromagnetic field distribution on the $\mathrm{MgO}$ substrate with the experimental dimensions and dielectric constants. The distribution of the x-axis electric field intensity on the substrate under the irradiation around the frequency $76 \mathrm{GHz}$ was obtained with the CST microwave studio software [Fig. 3(b)]. There was a resonant mode of the substrate was excited. However the electric field intensity was rather inhomogeneous along the grain boundary. The position of each subarray was marked by its order number in Fig. 3(b). Suppose that the $\Delta I_{\mathrm{c}} / I_{\mathrm{c}}$ of the subarray was in direct proportion to the coupled irradiation power, the implications from the Figs. 3(a) and 3(b) coincided with each other very well.

\section{B. Yttrium-stabilized zirconium}

The Josephson junction arrays located on a series of YSZ substrates with different thicknesses were investigated in order to achieve the optimal value for the coupling. Furthermore, in some cases the additional thin substrates of $\mathrm{MgO}$ and $\mathrm{LaAlO}$ were attached as well. All the significant measurements of the Shapiro steps and the self-emission from the total array are listed in Table I. For the Shapiro steps, we only list the parameters for the observed first Shapiro steps including the frequency of external irradiation and the maximum voltage of the first step which could be reached by increasing the number of biased subarrays. This suggested that the maximum voltage reflected the maximum number of coherent junctions inside the total array under external irradiation.

The junction arrays exhibited the distinct characters of the ac Josephson effects ${ }^{1}$ on the different substrates, especially for the $0.3-\mathrm{mm}$-thick substrate with and without the additional thin substrates. This reflected the important influence of the substrate on the impedance coupling, which could be considered as the dielectric resonator antenna. The resonance modes would change for different materials and dimensions of the substrate. This was the reason why the dissimilar Shapiro steps and self-emission power were observed even for the same array on different substrates. However, among all the samples with various substrates, the observed Shapiro steps and self-emission almost reached an optimum at the same time. It suggested that a proper substrate would improve both of these reversible processes.

A resonance mode of the 0.5 -mm-thick substrate ${ }^{8}$ was shown in Fig. 4. That was a reasonable explanation for the fact that the $85.06 \mathrm{mV}$ Shapiro steps and respectable emission power from the eight series subarrays were observed. The external irradiation coupled into each junction of the total array including 536 junctions should be approximately uniform. At present, we would like to concentrate more closely on the sample with the $0.3 \mathrm{~mm}$ thick substrate.

After attaching the additional thin substrate of LaAlO, the coupling of the junctions to the open space was obviously improved (Table I). The maximum voltage of the first Shapiro step could be increased from 46.92 to $84.29 \mathrm{mV}$. It indicated that there were more junctions in the series array realized coherence under external irradiation. At the same time, the irradiation frequency was shifted from 68.98 to $77.10 \mathrm{GHz}$, as shown in Fig. 5. Under external irradiation of other two frequencies, $72.85 \mathrm{GHz}$ and $75.00 \mathrm{GHz}$, the first Shapiro steps were also observed with the maximum voltages of $69.56 \mathrm{mV}$ and $82.16 \mathrm{mV}$, respectively. In addition,



FIG. 4. (Color online) Distribution of electric field intensity on the 0.5 -mmthick substrate shows a resonance mode around $77 \mathrm{GHz}$. 


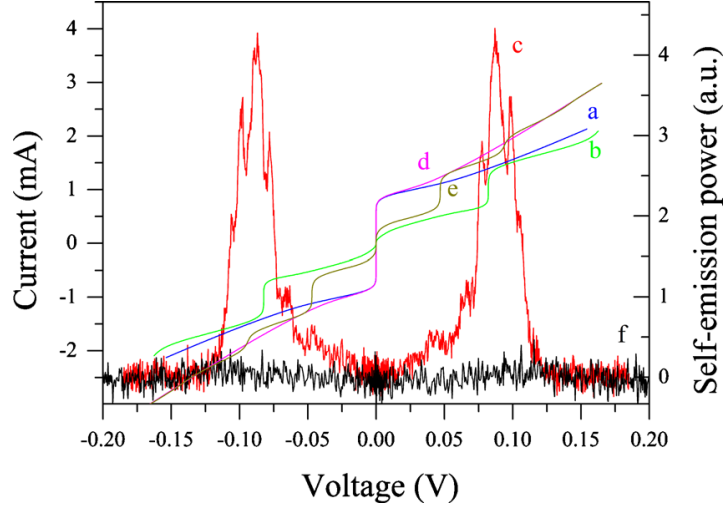

FIG. 5. (Color online) Measurements of the junction array located on the 0.3 mm thick YSZ substrate with and without the attached thin substrate of LaAlO. With the attached substrate, curve a shows the $I-V$ characteristic of the total series array including eight subarrays without irradiation; curve b: the $I-V$ characteristic under irradiation at $77.10 \mathrm{GHz}$; curve c: the detected emission power while sweeping the bias current through the array. Without the attached substrate, there was only a maximum of five biased subarrays from which the Shapiro steps could be observed. The $I-V$ curves of these five subarrays without and with irradiation at $68.98 \mathrm{GHz}$ are shown by curve $\mathrm{d}$ and curve e, respectively. The detected emission from the total array is showed by curve f.
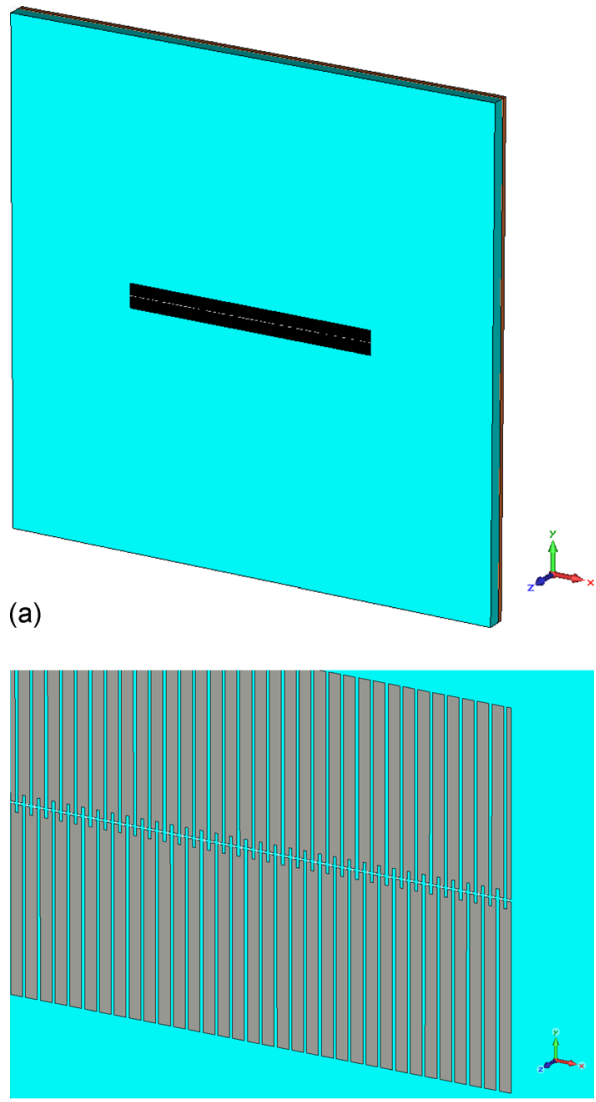

(b)

FIG. 6. (Color online) The simulation model for irradiation: the junction array (black color) with half-wavelength antennas was located on the YSZ substrate (blue color). The additional thin substrate of LaAlO (orange color) was attached on the backside of the YSZ substrate. The junction was modeled by the lumped element in the CST microwave studio software, by means of which the induced voltage of each junction could be observed. (a) Threedimensional view; (b) enlarged view.
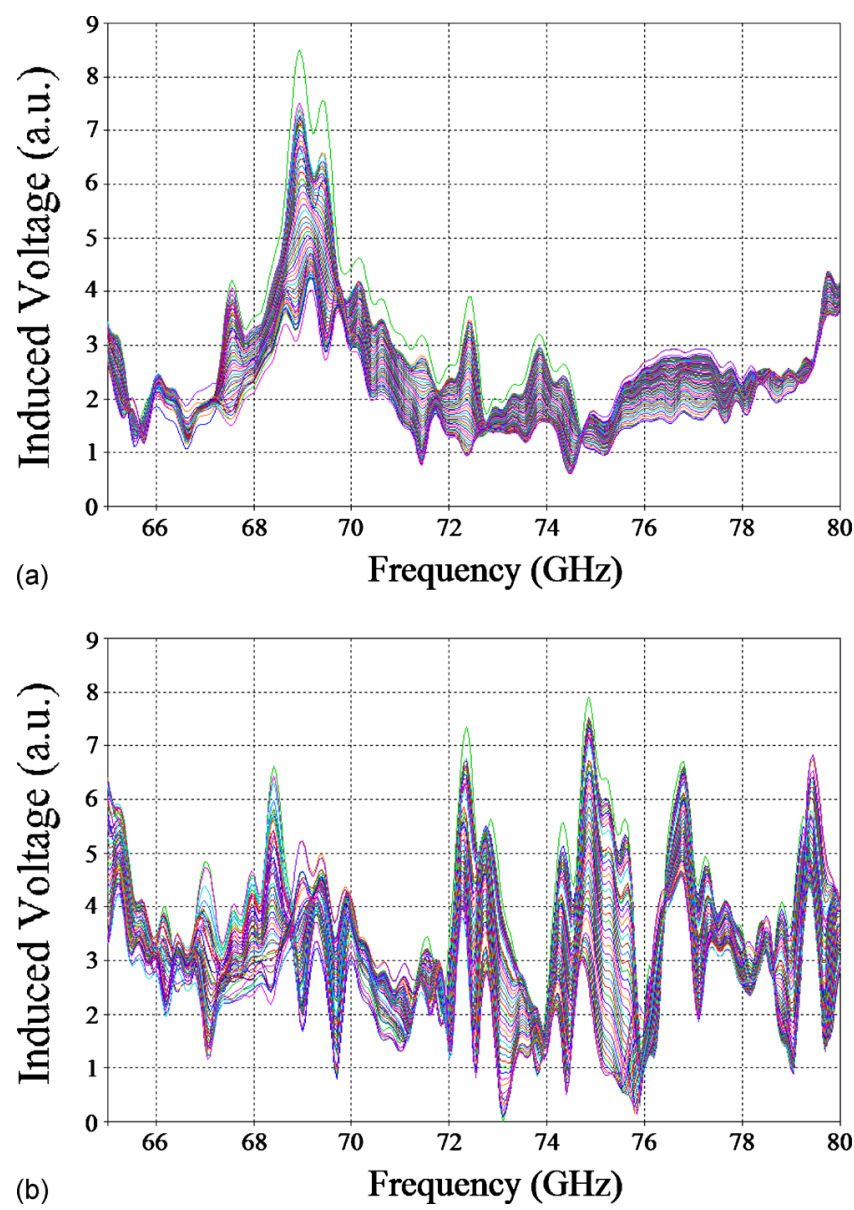

FIG. 7. (Color online) Induced voltage spectra of 50 junctions inside the total array from 65 to $80 \mathrm{GHz}$ without and with the attached substrate of LaAlO are shown separately in (a) and (b).

the self-emission power from the total array became more than ten times larger (Fig. 5). All the above phenomena agreed with our initial idea ${ }^{8}$ that a suitable junction substrate could be used as the dielectric resonator antenna. Furthermore the resonator allowed independent substrates of different materials to be combined together.

To analyze the experiments by simulating the electromagnetic field, a model was constructed of the junction arrays located on substrates under external irradiation from 65 to $80 \mathrm{GHz}$. In Fig. 6, the junctions were embedded in the half-wavelength antennas and connected in series as experimental parameters. ${ }^{8}$ The alternating voltage of each junction induced by the irradiation could be observed using this model. The value of the voltage was proportional to the square root of the irradiation power fed into the junctions. In this way, the Shapiro steps could only be observed in experiments when the induced voltage was uniform with acceptable amplitude for each junction. This has been proved by simulations as shown in Fig. 7. Limited by the resolution, induced voltages of 50 junctions in the total array were exhibited in this spectrum. For the 0.3 -mm-thick substrate, only one peak around $69 \mathrm{GHz}$ was obtained. While there were three uniform peaks in the frequency range from 68 to 78 $\mathrm{GHz}$ after the thin substrate of LaAlO had been attached. 
These peaks were located separately at about 73,75 , and 77 GHz. All of them agreed with the experiment results (Table I).

\section{CONCLUSIONS}

The same layout series array of bicrystal junctions was located on various substrates to investigate the impedance coupling of the junctions to the open space. The important influence of the substrates on the coupling was documented both by experiments and simulations. It is reasonable to consider the substrate as a dielectric resonator antenna to improve the coupling with suitable dimensions and materials. It was also possible to introduce the combination of substrates with different materials for some applications. It should be emphasized that this method could be utilized both for the development of voltage standards and for radiations sourced in the higher frequency range.

\section{ACKNOWLEDGMENTS}

The author Fengbin Song is supported by the State Scholarship Fund organized by CSC (China Scholarship Council).

${ }^{1}$ B. D. Josephson, Adv. Phys. 14, 419 (1965).

${ }^{2}$ A. K. Jain, K. K. Likharev, J. E. Lukens, and J. E. Sauvageau, Phys. Rep. 109, 309 (1984).

${ }^{3}$ Dielectric Resonator Antennas, edited by K.-M. Luk and K.-W. Leung (Research Studies, Hertfordshire, 2003).

${ }^{4}$ G. Kunkel and R. H. Ono, Appl. Phys. Lett. 69, 1960 (1996).

${ }^{5}$ P. A. A. Booi and S. P. Benz, Appl. Phys. Lett. 68, 3799 (1996).

${ }^{6}$ A. M. Klushin, M. He, S. L. Yan, and N. Klein, Appl. Phys. Lett. 89, 232505 (2006).

${ }^{7}$ F. Song, F. Muller, R. Behr, and A. M. Klushin, Appl. Phys. Lett. 95, 172501 (2009).

${ }^{8}$ F. Song, M. Y. Levitchev, V. A. Markelov, V. V. Kurin, L. Fang, and A. M. Klushin, Supercond. Sci. Technol. 23, 034026 (2010).

${ }^{9}$ C. A. Hamilton, Rev. Sci. Instrum. 71, 3611 (2000).

${ }^{10}$ B. Jeanneret and S. P. Benz, Eur. Phys. J. Spec. Top. 172, 181 (2009).

${ }^{11}$ M. I. Faley, S. B. Mi, A. Petraru, C. L. Jia, U. Poppe, and K. Urban, Appl. Phys. Lett. 89, 082507 (2006).

${ }^{12}$ A. M. Klushin, C. Weber, M. Darula, R. Semerad, W. Prusseit, H. Kohlstedt, and A. I. Braginski, Supercond. Sci. Technol. 11, 609 (1998). 